\title{
High redshift gamma-ray bursts
}

\author{
N. R. Tanvir ${ }^{* \dagger}$ \\ Department of Physics and Astronomy, University of Leicester, University Road, Leicester, LE1 \\ 7RH. United Kingdom \\ E-mail: nrt3@leicester.ac.uk
}

The brightest gamma-ray bursts (GRBs) can be seen at very high redshifts, offering a probe of star-formation and galaxy evolution, into the reionization era and beyond. Their bright afterglows pinpoint their host galaxies, however faint, and can give redshifts, metallicity estimates, information on the presence of dust and molecules, and HI column densities. Statistical samples of well-observed GRBs at high redshift may therefore tell us about the evolution of the global star formation rate, chemical enrichment, UV escape fraction and the galaxy luminosity function; all of which are very difficult to establish by conventional searches for galaxies. To date, only a handful of $z>6$ GRBs have been discovered, but their presence at $z>8$ begins to realise their potential as searchlights to illuminate the early Universe.

Gamma-Ray Bursts 2012 Conference-GRB2012,

May 07-11, 2012

Munich, Germany

\footnotetext{
* Speaker.

${ }^{\dagger}$ It is a pleasure to acknowledge my many collaborators who have contributed to various aspects of the work described here, particularly Andrew Levan and Klaas Wiersema.
} 


\section{Introduction}

By $z \sim 6$ the reservoir of gas between the nascent galaxies had become almost completely ionized [1]. This phase change presumably accompanied a rising far-ultraviolet and/or X-ray ionizing $[2,3]$ radiation field. It is frequently argued that this radiation was likely produced by early generations of massive stars, and hence that the era of reionization is intimately connected to the collapse and turning on of the first galaxies.

Unfortunately, sources at $z>7$ are very hard to study directly since, in addition to high luminosity distance, galaxies and quasars were both smaller and fainter at early times. Only with the re-observation of the Hubble Ultra-Deep Field HUDF with the Wide Field Camera-3 infrared channel, have significant numbers of candidate $z>7$ Lyman-break galaxies been identified for the first time [4-6].

Gamma-ray bursts offer another probe of the early Universe that is in many respects complementary to other methods. GRBs pinpoint their host galaxies and allow us to conduct a census of the locations and rates of massive star formation through cosmic history. Afterglow spectroscopy can reveal not only redshifts, but also detailed properties of the hosts such as chemical abundances [9-12], molecular content [13], dynamics, and dust properties [14-16]. Measurements of the red damping wing of the Ly $\alpha$ absorption line may provide both constraints on the neutral fraction of the intergalactic medium at the location of the burst [17-19] and the column of neutral ISM . Here I consider progress to-date in studying high redshift GRBs their hosts and afterglows, and consider prospects for the future.

\section{The search for high redshift GRBs}

In principle the brightest GRBs and their afterglows can be detected to very extreme redshifts, as is illustrated by Figure 1 which shows (in this case the the TOUGH sample) that some bursts would be above the effective sensitivity of Swift at least to $z \sim 10$.

The unpredictability and short timescale of the GRB phenomenon makes them challenging to observe, requiring wide field detectors and rapid response follow-up facilities. Obtaining redshifts for high- $z$ GRBs can be particularly problematic, usually requiring an initial indication of an optical dropout source, followed by near-IR spectroscopy. GRB 050904 was the first identified at $z>6$, being identified photometrically [20] and ultimately spectroscopically confirmed at $z=6.30$ [21]. This spectrum showed metal absorption lines giving an accurate redshift, and provided a weak constraint on the neutral fraction of the intergalactic medium [22]. Subsequently, GRB 080913 was found at $z=6.73[23,24]$ and also showed a single metal absorption line.

To date, the record spectroscopic redshift remains that of GRB 090423 at $z \approx 8.2[25,26]$. Unfortunately, in that case the afterglow was not bright enough, even at early times, to provide a high signal-to-noise spectrum, and so the redshift relied solely on the location of the Ly $\alpha$ break. Nevertheless, this event proved that GRBs were being produced at such early times in the universe, beyond any spectroscopically measured galaxy or QSO redshift.

An even more distant event may have been GRB 090429B, which had a best-fit redshift based on photometry of its afterglow of $z \approx 9.4$, however it was unfortunately not possible to obtain a spectrum of this event due to high winds at Mauna Kea which forced the closure of most tele- 


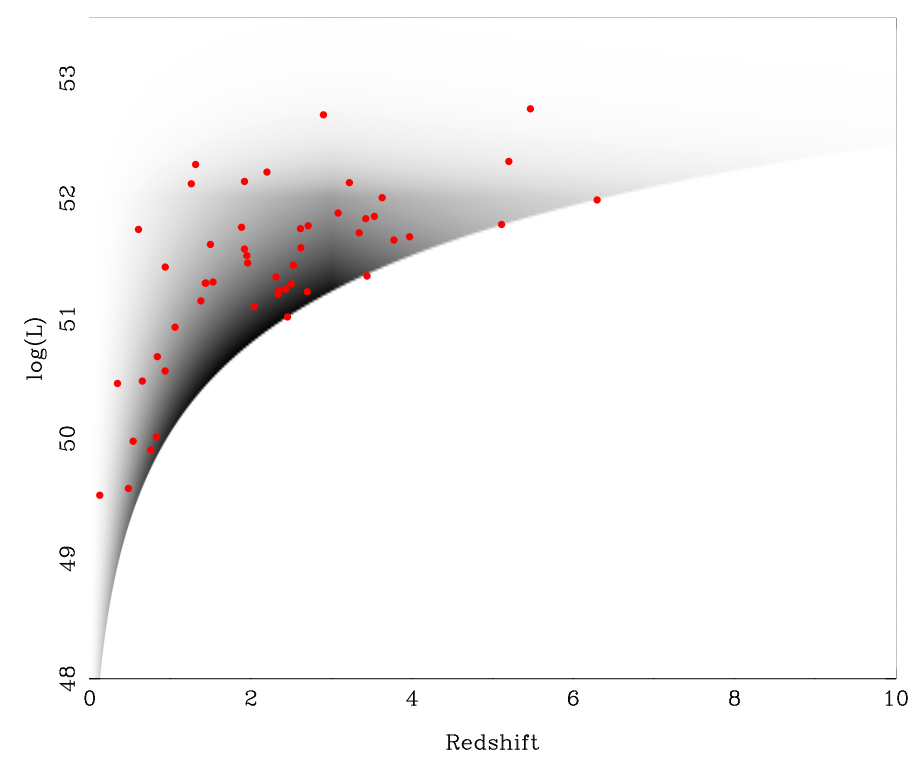

Figure 1: The base 10 logarithm of peak luminosity in units of $\mathrm{erg} \mathrm{s}^{-1}$ (1-s binning) against redshift for the GRBs in the TOUGH sample [7]. The grey shaded region shows the expected density of GRBs in this plane based on assuming they follow a metallicity-dependent star-formation rate history as described by [8], and with a non-evolving Schecter luminosity function. The selection function is indicated by the lower cut-off of the shaded region, although note that in practice the Swift detection threshold is not strictly a fixed peak luminosity. One implication of this figure is that the most luminous GRBs should certainly be detectable by Swift to $z>10$ (and in fact higher, since the search trigger actually looks for significant rate enhancements on a variety of timescales).

scopes. A lower redshift, down to about $z \sim 7$, would be allowed if there is a modest amount of rest-frame dust extinction [27] (see Figure 2). It is worth emphasising that photometric redshifts for GRBs tend to be more robust than for galaxies thanks to the simple underlying power-law continuum of their afterglows. A few other GRBs have $z \gtrsim 6$ based on either photometry or low-S/N spectroscopy [28], but the numbers remain small. Despite this, as many authors have pointed out, the rate of $z \gtrsim 4$ GRBs is considerably in excess of predictions based on the assumption that it should follow the canonical star-formation rate history (or even plausible estimates of the lowermetallicity star formation rate history) [30, 29, 8]. This could be because we do not understand the conditions required to produce GRBs from massive stars, or alternatively it could be indicating that traditional routes to estimating global star-formation via galaxy surveys are producing systematic underestimates at high- $z$, a topic we return to below.

\section{The hosts of high redshift GRBs}

A number of authors have recently emphasised that searches for high- $z$ GRBs hosts can potentially constrain the faint end of the galaxy luminosity function [31-33]. The hosts of $z>5$ GRBs have proven elusive. For example, both HST and Spitzer, finding no evidence of significant host emission in the field of GRB 050904 [34].

The locations of six $z>5$ GRBs have deep, late time HST infrared imaging (see Fig. 3). Only for GRB 060522 at $z=5.11$ is there marginal detection of flux close to the GRB location, whereas the data provide deep limits on any host contribution in the other cases. We note that even few orbit 

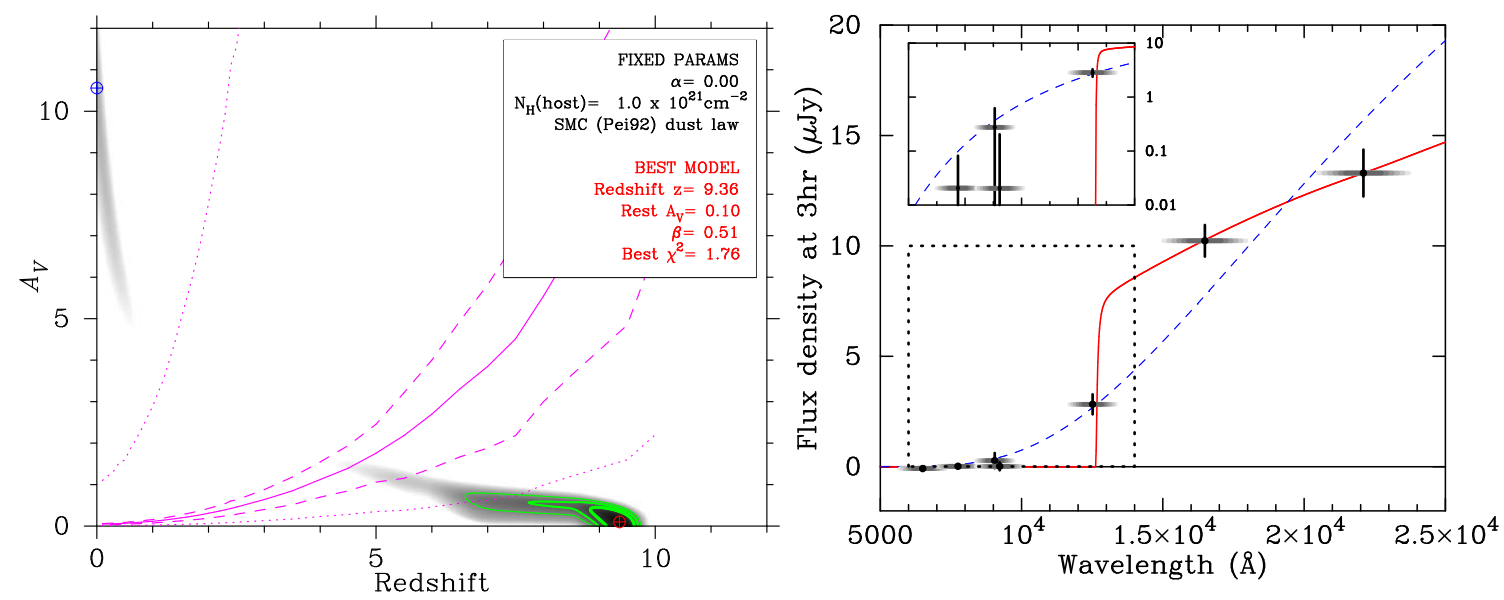

Figure 2: The photometric redshift estimate for GRB 090429B. [Left panel:] shows likelihood contours in a plane of redshift versus rest-frame extinction obtained by fitting an intrinsic power-law spectral energy distribution with dust attenuation (in this case assumed to follow an SMC dust law, which has commonly been found for lower-redshift GRBs), to the observed afterglow photometry. The three (green) contours overlaying the greyscale represent $90 \%, 99 \%$ and 99.9\% confidence respectively. The most likely value of the redshift is found to be $z \approx 9.4$. [Right panel:] the bold red line shows the best fitting model, and the solid vertical bars the error ranges for the photometry. The horizontal bars are not errors but simply indicate the approximate widths of the broad-band filters used. The best-fit low redshift solution is shown as a dashed blue curve, and is formally ruled out at high significance. (The inset shows the dashed region but with a logarithmic flux axis).

integrations can provide deep constraints since, unlike galaxies found in deep field samples which require relatively high-S/N detections and multiple bands to perform photometric redshift analysis, for the GRB hosts we can accept even $2 \sigma$ detections, for example, as meaningful, given we know both redshift and position in advance.

The key question is whether the data are consistent with a steepening faint-end slope as indicated by some analyses of the UDF. The likelihood of the GRB hosts being as faint as observed can be assessed by taking the galaxy UV luminosity functions estimated from HST deep field observations, weighting these by luminosity (thus providing a probability density function for the host galaxy luminosities, assuming GRB rate is proportional to star-formation rate and hence proportional to UV luminosity), and finally convolving with observational errors appropriate to each dataset. This should provide a prediction for probability density function for the observed flux at each host location, which can be compared to the results from the images themselves.

Such an analysis indeed agrees with the suggestion that the bulk of star formation at $z>6$ is occurring in small, faint galaxies below the detection level of the HUDF [31]. With the present sample this is only sufficient to marginally reject a non-evolving LF shape (at the $\sim 90 \%$ level), however it does illustrate the key role GRBs may play in the future study of star formation in the era of reionization, giving access to the dominant population of star-forming galaxies.

\section{Future prospects}

In principle modelling the red damping wing of the Ly $\alpha$ absorption trough can distinguish absorption due to the IGM from features due to the host galaxy. In practice, this will frequently be difficult due to the large neutral gas columns often found in GRB host galaxies [35, 36], and the 


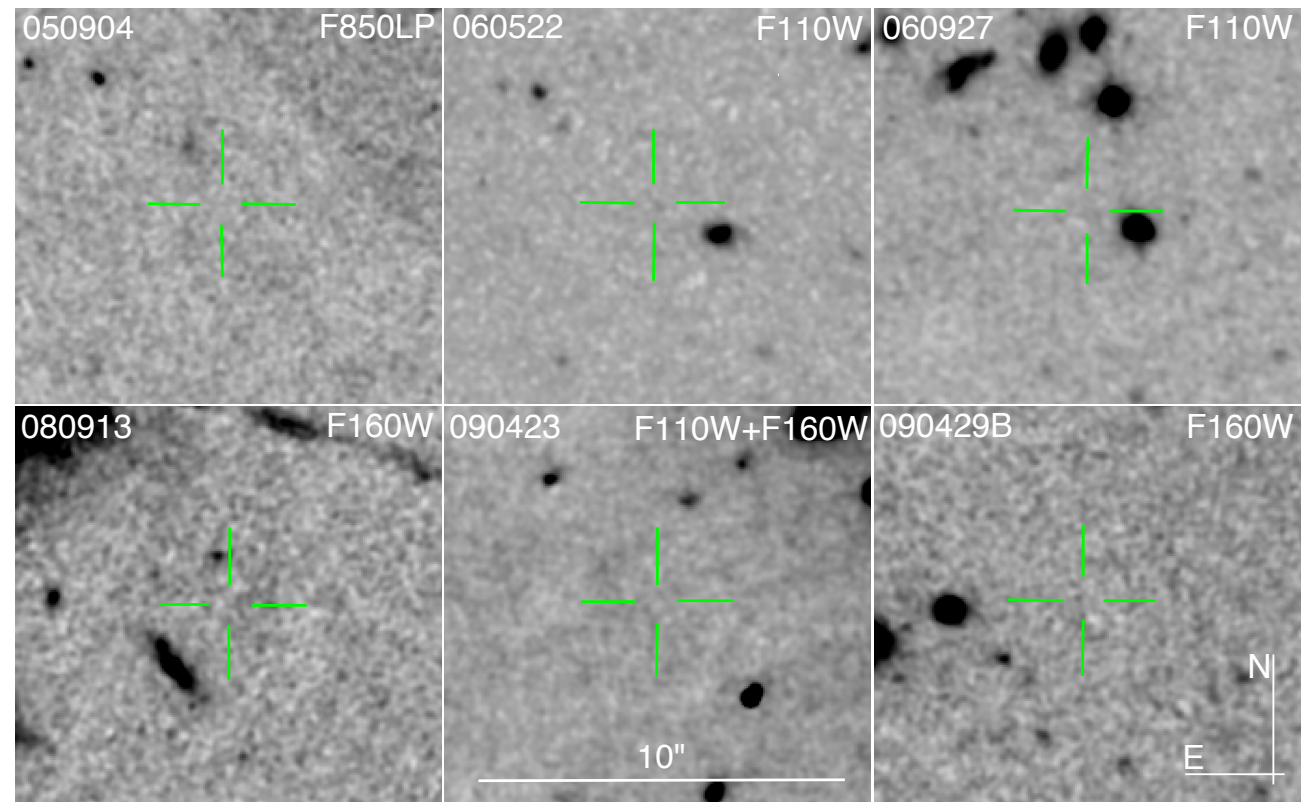

Figure 3: Mosaic of HST infrared images (corresponding to rest-frame UV) for the locations of six $z>5$ GRBs [31]. The images were all obtained months or years post-burst when the contribution from afterglow light should be negligible. Only for GRB 060522 at $z=5.11$ is there marginal evidence for flux at the location of the burst (indicated by cross-hairs) while in no other case is there a significant $(>2 \sigma)$ detection. This finding supports the idea that the majority of star formation at high redshifts is occurring in galaxies below deep HST detection limits.

possibility that they (and their neighbours) also produce other effects on the shape of the absorption profile, in particular by ionizing some region around the galaxy. It is likely that very high signalto-noise spectra will be required to confidently disentangle these components in most cases. Such spectra could be acquired with present technology for unusually bright afterglows, but more likely will have to await larger future telescopes. In Fig. 4 I show a simulation of an afterglow spectrum (in fact based on the parameters of GRB 090423) as it could have been seen with a next-generation ground-based telescope like the proposed European Extremely Large Telescope (E-ELT). The quality of the spectra is easily sufficient to accurately measure the neutral fraction of the IGM if the gas column in the host is small. For larger host columns this will be more difficult (although useful results may still be obtained with reduced accuracy), but in compensation such systems will allow abundance measurements from metal absorption features down to very low metallicities.

Even with such observations, as emphasised by [19], the expected variance from sight-line to sight-line of the neutral fraction at any given redshift, will mean that many high- $z$ GRBs would need to be observed in order to provide a detailed timeline of reionization and an indication of its topology.

However, the same observations would also provide us with estimates of the neutral hydrogen column in the ISM of the hosts. This is potentially important since the column can be translated into an optical depth to ionizing radiation $[36,37]$. Thus a sample of such measures would allow us to 
$z=8.2$ simulated ELT afterglow spectrum

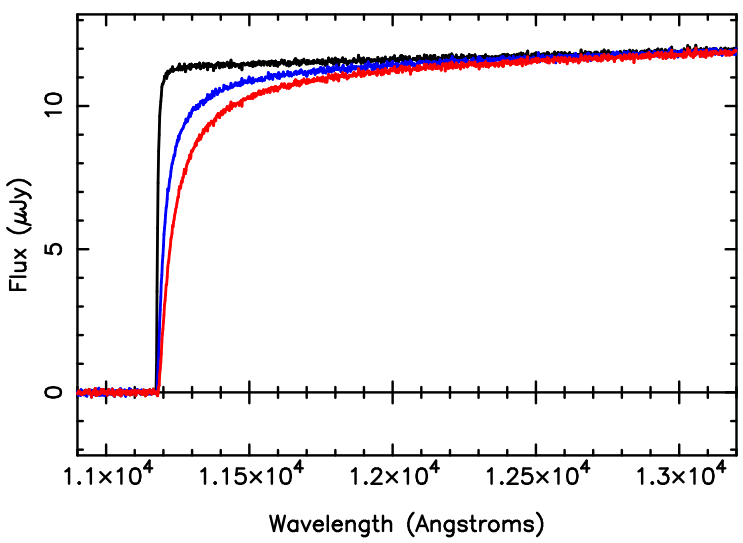

$z=8.2$ simulated ELT afterglow spectrum

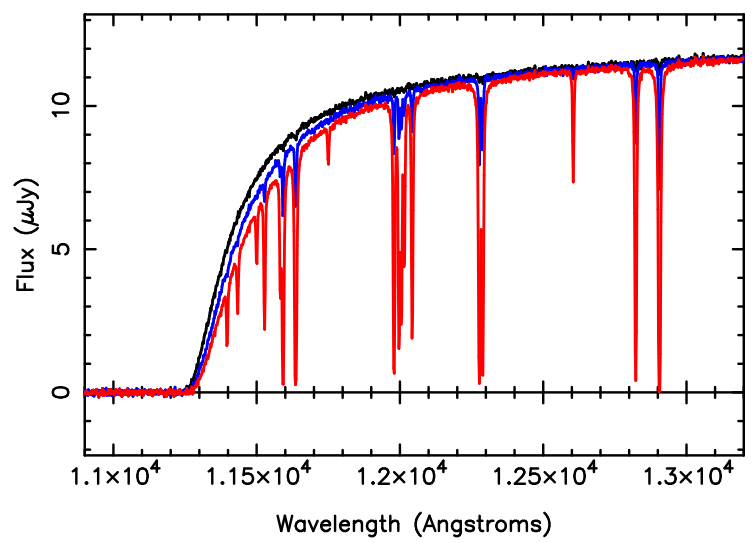

Figure 4: Simulated spectra showing the quality of data which would be obtained with a $\sim 40 \mathrm{~m}$ telescope such as the proposed E-ELT for an afterglow with magnitude approximately the same as that obtained for GRB 090423 observed by the VLT [25]. [Left panel:] is for the situation of very low HI column within the host galaxy, and the three curves show the effect of an IGM neutral fraction of $0 \%, 50 \%$ and $100 \%$ from top to bottom. [Right panel:] in the case where the host itself has a high column density of HI, it will be harder to measure the IGM neutral fraction but much easier to measure abundances in the host. The particular simulation has $N_{\mathrm{H} \text {,host }}=10^{22} \mathrm{~cm}^{-2}$ (offset slightly between models for clarity), and metallicity ranging through $0.1 \%, 1 \%$ and $10 \%$ of Solar from top to bottom.

gauge the escape fraction of UV continuum, which is essentially for understanding the contribution of massive stars to reionization.

\section{Conclusions}

Thanks to the brightness of the prompt and afterglow emission of long-duration GRBs, they offer a powerful probe of star formation and galaxy evolution in the early universe. They provide us with a means of studying massive star formation, its rate, location and environments, in principle even when it is taking place in galaxies beyond the detection limit of current or planned future telescopes. If the evolution of the galaxy luminosity function is proceeding as rapidly as some studies suggest, then such, very faint galaxies could dominate the total star formation activity in the peak era of reionization, and at even earlier times. This is consistent with the non-detection by $H S T$ of the highest redshift GRB hosts, and strongly motivates the development of new missions which are capable of finding significant numbers of GRBs at extreme redshifts.

\section{References}

[1] S. Malhotra, J. E. Rhoads, Luminosity functions of Lyman- $\alpha$ emitters at Redshift $z=6.5$ and $z=5.7$ : Evidence against reionization at $z \approx 6$, ApJ 617 (2004) L5

[2] A. Loeb The Race Between Stars and Quasars in Reionizing Cosmic Hydrogen, JCAP 3 (2009) 22

[3] I. F. Mirabel, M. Dijkstra, P. Laurent, A. Loeb, J. R. Pritchard, Stellar black holes at the dawn of the universe, A\&A $\mathbf{5 2 8}$ (2011) A149

[4] A. J. Bunker et al., The Contribution of High Redshift Galaxies to Cosmic Reionization: New Results from Deep WFC3 Imaging of the Hubble Ultra Deep Field, MNRAS 409 (2010) 855 
[5] R. J. McLure et al., Galaxies at z =6-9 from the WFC3/IR imaging of the HUDF, MNRAS 403 (2010) 960

[6] R. J. Bouwens et al., Ultraviolet Luminosity Functions from $132 z \sim 7$ and $z \sim 8$ Lyman-break Galaxies in the Ultra-deep HUDF09 and Wide-area Early Release Science WFC3/IR Observations, ApJ 737 (2011a) 90

[7] J. Hjorth et al., The optically unbiased GRB host (TOUGH) survey. I. Survey design and catalogs, [arXiv:1205.3161]

[8] P. Jakobsson et al., The optically unbiased GRB host (TOUGH) survey. III. Redshift distribution, ApJ 752 (2012) 62

[9] Vreeswijk P. M., et al., The host of GRB 030323 at $z=3.372$ : A very high column density DLA system with a low metallicity, A\&A 419 (2004) 927

[10] H.-W. Chen, J. X. Prochaska, J. S. Bloom, I. B. Thompson, Echelle Spectroscopy of a Gamma-Ray Burst Afterglow at $z=3.969$ : A New Probe of the Interstellar and Intergalactic Media in the Young Universe, ApJ 634 (2005) L25

[11] J. P. U. Fynbo et al., Probing cosmic chemical evolution with gamma-ray bursts: GRB 060206 at $z=4.048, A \& A 451$ (2006) L47

[12] J. X. Prochaska et al., Probing the Interstellar Medium near Star-forming Regions with Gamma-Ray Burst Afterglow Spectroscopy: Gas, Metals, and Dust, ApJ 666 (2007) 267

[13] J. X. Prochaska et al., The First Positive Detection of Molecular Gas in a GRB Host Galaxy, ApJ 691 (2009) L27

[14] P. Schady et al., Dust and metal column densities in gamma-ray burst host galaxies, MNRAS 401 (2010) 2773

[15] Zafar T., et al., The extinction curves of star-forming regions from $z=0.1$ to 6.7 using GRB afterglow spectroscopy, A\&A 532 (2011) A143

[16] Zafar T., et al., Exploring Dust Extinction at the Edge of Reionization, ApJ 735 (2011) 2

[17] J. Miralda-Escude, Reionization of the Intergalactic Medium and the Damping Wing of the Gunn-Peterson Trough, ApJ 501 (1998) 15

[18] R. Barkana R., A. Loeb, Gamma-ray bursts versus quasars: Lyman- $\alpha$ signatures of reionization versus cosmological infall, ApJ 601 (2004) 64

[19] M. McQuinn et al., Probing the neutral fraction of the IGM with GRBs during the epoch of reionization, MNRAS 388 (2008) 1101

[20] J. B. Haislip et al., A photometric redshift of $z=6.39 \pm 0.12$ for GRB 050904, Nature 440 (2006) 181

[21] N. Kawai et al., An optical spectrum of the afterglow of a gamma-ray burst at a redshift of $z=6.295$, Nature 440 (2006) 184

[22] T. Totani et al., Implications for Cosmic Reionization from the Optical Afterglow Spectrum of the Gamma-Ray Burst 050904 at $z=6.3$, PASJ 58 (2006) 485

[23] J. Greiner et al., GRB 080913 at Redshift 6.7, ApJ 693 (2009) 1610

[24] M. Patel, S. J. Warren, D. J. Mortlock, J. P. U. Fynbo, The reanalysis of spectra of GRB 080913 to estimate the neutral fraction of the IGM at a redshift of 6.7, A\&A 512 (2010) L3

[25] N. R. Tanvir et al., A gamma-ray burst at a redshift of $z \sim 8.2$, Nature 461 (2009) 1254 
[26] R. Salvaterra et al., GRB090423 at a redshift of $z \sim 8.1$, Nature 461 (2009) 1258

[27] A. Cucchiara et al., A Photometric Redshift of $z \sim 9.4$ for GRB 090429B, ApJ 736 (2011) 7

[28] N. R. Tanvir et al., GRB 120521C redshift estimate, GCN Circular 13348 (2012)

[29] B. E. Robertson and R. S. Ellis, Connecting the gamma ray burst rate and the cosmic star formation history: implications for reionization and galaxy evolution, ApJ 744 (2012) 95

[30] H. Yüksel et al., Revealing the high-redshift star formation rate with gamma-ray bursts, ApJ $\mathbf{6 8 3}$ (2008) L5

[31] N. R. Tanvir et al., Star formation in the early universe: beyond the tip of the iceberg, ApJ in press, [arXiv:1201.6074]

[32] M. Trenti et al., Gamma-Ray Burst Host Galaxy Surveys at Redshift z > 4: Probes of Star Formation Rate and Cosmic Reionization, ApJ 749 (2012) L38

[33] S. Basa et al., Constraining the nature of the most distant Gamma-Ray Burst host galaxies, [arXiv:1201.6383]

[34] E. Berger et al., Hubble Space Telescope and Spitzer Observations of the Afterglow and Host Galaxy of GRB 050904 at $z=6.295$, ApJ 665 (2007) 102

[35] P. Jakobsson et al., A mean redshift of 2.8 for Swift gamma-ray bursts, A\&A 460 (2006) L13

[36] H.-W. Chen, J. X. Prochaska, N. Y. Gnedin, A New Constraint on the Escape Fraction in Distant Galaxies Using Gamma-Ray Burst Afterglow Spectroscopy, ApJ 667 (2007) L125

[37] J. P. U. Fynbo et al., Low-resolution spectroscopy of gamma-ray burst optical afterglows: biases in the swift sample and characterization of the absorbers, ApJS 185 (2009) 526 